ESAIM: PROCEEDINGS, May 2009, Vol. 27, p. 156-170

C. Besse, O. Goubet, T. Goudon \& S. Nicaise, Editors

\title{
UTILISATION DES ÉQUATIONS EULER-PML EN MILIEU HÉTÉROGÈNE BORNÉ POUR LA RÉSOLUTION D'UN PROBLÈME INVERSE EN GÉOPHYSIQUE.
}

\author{
Ludovic MÉTIVIER ${ }^{1}$
}

\begin{abstract}
The numerical resolution of a seismic non linear inverse problem leads to use 2D EulerPML equations, as a model of acoustic wave propagation in the underground. The numerical domain where the computation is done is bounded and heterogeneous. Some a priori energy estimates are proposed in order to demonstrate that this propagation problem is weakly well-posed with a default of rank 1, as an extension of the results in [Petit-Bergez, 2006].

Résumé. La résolution d'un problème non linéaire d'inversion de données sismiques donne lieu à l'utilisation d'équations 2D Euler-PML pour la modélisation de la propagation des ondes. Le domaine numérique de calcul est borné et hétérogène. Des estimations d'énergie a priori sont proposées; elles démontrent notamment, en étendant les résultats de [Petit-Bergez, 2006], que le problème est faiblement bien posé, de défaut 1.
\end{abstract}

\section{INTRODUCTION}

L'inversion de données sismiques est une figure emblématique de la famille des problèmes inverses. A la suite de l'explosion d'une source en surface, des récepteurs enregistrent les réflexions des ondes émises sur les discontinuités du sous-sol. Ces enregistrements constituent les données à partir desquelles une estimation des paramètres élastiques du sous-sol, à l'origine des réflexions, est recherchée. Les applications de cette technique sont diverses, telles la prospection pétrolière ou l'ingénierie civile. Les méthodes actuellement employées fournissent un résultat d'une précision de l'ordre de la dizaine de mètres. Cependant, l'intérêt des industriels se porte sur l'obtention d'une information de plus en plus précise, et une description du sous-sol à l'échelle du mètre, voire inférieure, est recherchée, notamment pour la problématique du stockage de CO2. De plus, les milieux de propagation fortement hétérogènes (variations de l'ordre de la dizaine de centimètres sur une profondeur de plusieurs centaines de mètres) perturbent fortement les méthodes classiques qui mènent alors à des résultats erronés. Une possibilité de s'affranchir de cette difficulté, et parvenir à des résultats dits de "haute résolution", est la prise en compte des non-linéarités de la propagation des ondes par rapport aux paramètres élastiques du sous-sol [Delprat-Jannaud, Lailly, 2004]. En effet, les méthodes usuelles utilisent des modèles de propagation linéaires qui ne prennent en compte que les réflexions primaires sur les discontinuités du sous-sol. Ceci explique notamment leur échec dans le cas de milieux fortement hétérogènes, puisque dans ce contexte les réflexions secondaires (ou scattering multiple) jouent un rôle qui ne peut plus être négligé. La prise en compte de ces non-linéarités induit cependant une sensibilité très forte par rapport à la source qui génère les données [Delprat-Jannaud, Lailly, 2005]. Or, celle-ci est en général assez mal connue, en raison des effets de

\footnotetext{
1 Université Paris 13, 99 Avenue J.B Carnot, 93500 Villetaneuse, Institut Français du Pétrole, 1-4 Avenue du Bois Préau, 92500 Rueil-Malmaison
}

(c) EDP Sciences, SMAI 2009 Article published by EDP Sciences and available at http://www.edpsciences.org/proc or http://dx.doi.org/10.1051/proc/2009025 
couplage entre la source et le sous-sol. Ceci constitue une difficulté majeure pour l'introduction d'un modèle de propagation non linéaire dans les méthodes d'inversion classiques.

Il devient alors intéressant de se pencher sur le cas particulier de l'inversion de données sismiques de puits. Contrairement à l'inversion de données sismiques de surface, dans laquelle les récepteurs enregistrant la réponse du sous-sol sont placés en surface, à une certaine distance ("offset") de la source, les récepteurs sont placés dans un puits vertical, à différentes profondeurs. Au cours de l'inversion, en plus du modèle d'impédance, la condition de pression au récepteur le moins profond est calculée. Cette condition de pression correspond au signal émis par la source après la traversée de l' "encaissant", c'est à dire l'épaisseur de terre qui sépare la source du premier récepteur. Cet intermédiaire de calcul permet de s'affranchir de la connaissance de la source a priori requise par les méthodes classiques. Le problème de sensibilité par rapport à la source est donc levé, et les non-linéarités de la propagation peuvent être prises en compte, conduisant au résultat haute résolution désiré. La difficulté réside ailleurs : l'étude et la mise en oeuvre de ce procédé n'ont été effectuées que dans un contexte de propagation et de description du sous-sol 1D [Macé, Lailly, 1986], s'inspirant de résultats mathématiques obtenus pour l'inversion de données sismiques en contexte 1D ( [Bamberger, 1978], [Bamberger, Chavent, Lailly, 1979]). Les limitations sont donc nombreuses. D'une part le modèle de sous-sol est 1D, et les paramètres ne peuvent être estimés que dans l'axe du puits. D'autre part, le modèle de propagation considéré suppose la propagation d'ondes planes, ce qui constitue une hypothèse fort peu réaliste. Pour s'affranchir de ces hypothèses restrictives, il est intéressant d'étudier l'extension de l'inversion de données de puits à un contexte multi-dimensionnel. L'application de cette méthode est donc étudiée dans un contexte 2D.

Le principe de résolution demeure inchangé : le problème est résolu par la minimisation d'une fonctionnelle mesurant l'écart quadratique entre les données observées et les résultats obtenus par la résolution du problème direct de propagation d'ondes. Cependant, le modèle d'impédance déterminé est maintenant 2D, et la condition de pression au premier récepteur ne dépend plus uniquement du temps, mais également de l'extension latérale choisie pour le modèle d'impédance. De plus, le modèle de propagation doit faire intervenir des conditions absorbantes afin de borner numériquement le domaine de calcul. Des couches absorbantes de type PML (Perfectly Matched Layers), qui s'appliquent aux systèmes hyperboliques, ont été choisies; de ce fait, la propagation des ondes acoustiques est décrite par le système des équations d'Euler 2D linéarisées autour d'une vitesse nulle. Le gradient de la fonctionnelle est calculé dans un cadre discret, par la méthode de l'état adjoint. Par ailleurs, la stratégie d'optimisation doit être adaptée à la grande taille du problème. Finalement, le nombre d'inconnues étant largement augmenté, il convient de déterminer quelle information a priori doit être introduite pour lever le maximum d'indétermination. Les problèmes posés sont donc nombreux. Il sera question ici plus particulièrement de l'étude mathématique du premier d'entre eux, à savoir la formulation et la démonstration du caractère faiblement bien posé du problème de propagation d'ondes dans un milieu 2D borné, hétérogène, bordé de couches absorbantes PML. Ces résultats sont déjà connus pour le problème de Cauchy en milieu homogène, pour les équations de Maxwell TE (Transverse Electrique) [Petit-Bergez, 2006]. La nouveauté proposée ici est une formulation adaptée à un problème de propagation 2D en milieu hétérogène, et le calcul d'estimations d'énergie pour la propagation d'ondes en milieu borné, hétérogène, les paramètres physiques du milieu pouvant présenter des discontinuités. La première partie sera consacrée à la formulation mathématique du problème inverse d'identification de l'impédance. Dans la deuxième partie seront présentés les résultats mathématiques obtenus pour le problème direct de propagation des ondes, à savoir les estimations d'énergie et la construction de ces résultats. 


\section{Description de la méthode D'inversion 2D De données sismiques de puits}

\subsection{Problème direct}

Les équations retenues pour modéliser la propagation d'ondes acoustiques sont les équations d'Euler 2D linéarisées autour d'une vitesse nulle,

$$
\left\{\begin{array}{l}
\partial_{t} u_{x}-\alpha c \partial_{x} p=0 \\
\partial_{t} u_{z}-\alpha c \partial_{z} p=0 \\
\partial_{t} p-\frac{c}{\alpha} \partial_{x} u_{x}-\frac{c}{\alpha} \partial_{z} u_{z}=0
\end{array}\right.
$$

avec des condition initiales nulles pour $p, u_{x}, u_{z}$, et la condition limite

$$
p\left(x, z_{1}, t\right)=h(x, t), \quad \text { avec } \quad h(x, 0)=0,
$$

où $u_{x}, u_{z}, p$ sont respectivement les vitesses de déplacement horizontal, vertical et la pression, définies sur $[a, b] \times\left[z_{1}, z_{\max }\right] \times[0, T]$. De plus,

$$
c \in C^{\infty}\left([a, b] \times\left[z_{1}, z_{\max }\right]\right), \quad \text { et } \alpha \in C_{p m}^{\infty}\left([a, b] \times\left[z_{1}, z_{\max }\right]\right)
$$

sont respectivement la vitesse des ondes acoustiques et l'impédance du milieu de propagation, $C_{p m}^{\infty}([a, b] \times$ $\left[z_{1}, z_{\max }\right]$ ) désignant l'ensemble des fonctions $C^{\infty}$ par morceaux sur le domaine $[a, b] \times\left[z_{1}, z_{\max }\right]$. En effet, si la vitesse peut être considérée comme une fonction lisse du domaine, l'impédance présente des discontinuités qui sont à l'origine des réflexions observées lors de la propagation des ondes dans le milieu. Pour le problème traité ici, la vitesse $c(x, z)$ est considérée connue. Le paramètre clef est donc l'impédance $\alpha(x, z)$, dont une estimation est recherchée lors de la résolution du problème inverse.

Des récepteurs sont disposés à intervalle régulier dans le puits. Leurs coordonnées sont notées

$$
\left(x_{r}, z_{r}\right), \quad 1 \leq r \leq R \text {. }
$$

Ces récepteurs mesurent les vitesses de déplacement horizontal et vertical. Ceci permet de définir les observations $O_{r}^{x}(t), O_{r}^{z}(t), \in L^{2}([0, T])$

$$
\forall t \in[0, T], \quad \forall r=1 \ldots R\left\{\begin{array}{l}
O_{r}^{x}(t)=u_{x}\left(x_{r}, z_{r}, t\right) \\
O_{r}^{z}(t)=u_{z}\left(x_{r}, z_{r}, t\right) .
\end{array}\right.
$$

Le problème direct est donc défini par l'opérateur de modélisation $\mathbf{M}$ qui, à un modèle d'impédance $\alpha(x, z)$ et une condition de pression $h(x, t)$, associe les valeurs du champ de vitesse au niveau des récepteurs, obtenues par la résolution du système (1), (2)

$$
\begin{gathered}
\mathbf{M}:(\alpha, h) \longmapsto\left(u_{x}\left(x_{1}, z_{1}, t\right), \ldots, u_{x}\left(x_{R}, z_{R}, t\right), u_{z}\left(x_{1}, z_{1}, t\right), \ldots, u_{z}\left(x_{R}, z_{R}, t\right)\right) \\
C_{p m}^{\infty}\left([a, b] \times\left[z_{1}, z_{\text {max }}\right] ; \mathbb{R}_{+}^{*}\right) \times L^{2}([a, b] \times[0, T]) \longmapsto\left(L^{2}([0, T])\right)^{2 R} .
\end{gathered}
$$




\subsection{Problème inverse}

L'opérateur inverse $\mathbf{M}^{-1}$ n'est pas défini. En effet, ni l'existence, ni l'unicité d'un modèle d'impédance et d'une condition de pression reproduisant exactement les données ne sont assurées. Il est donc nécessaire de construire un critère de sélection afin de définir une solution approchée du problème. Ce critère consiste en la mesure de l'écart quadratique entre les données et les observations calculées par la résolution du problème direct, pour un modèle d'impédance et une condition de pression donnés. La résolution du problème inverse consiste en la minimisation de ce critère [Bonnet, 2006]. Le problème inverse reste cependant, de par l'introduction massive d'inconnues supplémentaires par rapport au problème 1D, largement indéterminé, différents modèles pouvant aboutir au même écart quadratique. Afin de restreindre cette indétermination, il convient de réaliser plusieurs "tirs", correspondant à des positions de sources différentes, ceci afin de constituer un ensemble plus vaste de données. A chacun de ces jeux de données correspond une condition de pression à la frontière $z=z_{1}$, reconstituée au cours de l'inversion. Il faut donc considérer $N$ jeux de données correspondant à $N$ sources ponctuelles, auxquels sont associées $N$ conditions de pression $h_{n}(x, t)$. De plus, il existe plusieurs méthodes dites de "régularisation" pouvant être utilisées afin de tirer profit de toute l'information a priori disponible sur le modèle d'impédance recherché. Une description exhaustive de ces méthodes dépassant le cadre de cet exposé, la fonctionnelle considérée ici ne fera intervenir aucun critère de régularisation. Soit

$$
h=\left[\begin{array}{c}
h_{1}(x, t) \\
\vdots \\
h_{N}(x, t)
\end{array}\right] .
$$

La fonctionnelle à minimiser pour la résolution du problème inverse est

$$
J(\alpha, h)=\sum_{n=1}^{N} \sum_{r=1}^{R} \int_{0}^{T}\left|O_{r, n}^{x}(t)-\mathbf{M}_{r}\left(\alpha, h_{n}\right)(t)\right|^{2}+\left|O_{r, n}^{z}(t)-\mathbf{M}_{r+R}\left(\alpha, h_{n}\right)(t)\right|^{2} d t .
$$

Le problème inverse s'écrit alors

$$
\begin{gathered}
\min _{\alpha, h} J(\alpha, h) \\
\text { sous la contrainte } \quad \forall(x, z) \in[a, b] \times\left[z_{1}, z_{\max }\right], \quad \alpha(x, z)>0
\end{gathered}
$$

\section{Des couches absorbantes PML pour la RÉSolution du Problème direct}

\subsection{Formulation PML}

Pour pouvoir borner numériquement le domaine de calcul sans générer de réflexion artificielle, des couches absorbantes sont introduites. La formulation retenue est une adaptation aux équations d'Euler en milieu hétérogène de celle proposée dans [Bérenger,1993] pour les équations de Maxwell TE en milieu homogène. La multiplication des coefficients d'absorption $\sigma_{x}(x)$ et $\sigma_{z}(z)$ par la vitesse $c$ constitue la seule modification notable.

$$
\left\{\begin{array}{l}
\partial_{t} u_{x}-\alpha c \partial_{x} p_{x}-\alpha c \partial_{x} p_{z}+c \sigma_{x} u_{x}=0 \\
\partial_{t} u_{z}-\alpha c \partial_{z} p_{x}-\alpha c \partial_{z} p_{z}+c \sigma_{z} u_{z}=0 \\
\partial_{t} p_{x}-\frac{c}{\alpha} \partial_{x} u_{x}+c \sigma_{x} p_{x}=0 \\
\partial_{t} p_{z}-\frac{c}{\alpha} \partial_{z} u_{z}+c \sigma_{z} p_{z}=0
\end{array}\right.
$$


Les variables $p_{x}$ et $p_{z}$ sont issues du "splitting" de la variable de pression, et vérifient $p_{x}+p_{z}=p$. Les conditions initiales sont nulles pour $u_{x}, u_{z}, p_{x}, p_{z}$. La condition limite en $z=z_{1}$ est

$$
p_{x}\left(x, z_{1}, t\right)+p_{z}\left(x, z_{1}, t\right)=h(x, t)
$$

Il a été montré que le problème de Cauchy pour les équations de Maxwell TE PML en milieu homogène est faiblement bien posé de défaut 1 [Abarbanel, Gottlieb, 1997] [Bécache, Joly, 2002]. L'objectif est d'étendre ce résultat au problème aux limites pour les équations d'Euler PML en milieu hétérogène et d'obtenir des estimations d'énergie sur la solution. Pour simplifier la preuve, le problème est décomposé en deux parties. La première traite du problème aux limites en milieu hétérogène lisse, dans le cas simplifié du demi-espace $\left(\alpha \in C^{\infty}\left(\left[z_{1},+\infty\right] \times \mathbb{R}_{+} ; \mathbb{R}_{+}^{*}\right)\right)$. La condition limite en pression portera alors sur la frontière du demi-espace en $z=0$. La deuxième partie s'attache à l'étude du problème en milieu non borné, pour un milieu stratifié ( $\alpha$ ne dépend que de la direction $z$ ), pour une impédance présentant une discontinuité à l'interface entre deux sous-domaines $\left(\alpha \in C_{p m}^{\infty}\left(\mathbb{R} ; \mathbb{R}_{+}^{*}\right)\right)$. La conjonction des deux résultats obtenus montre le caractère faiblement bien posé du problème globale dans le cas d'un milieu de propagation stratifié : équations PML en milieu borné hétérogène avec de possibles discontinuités d'impédance suivant $z$. L'extension au cas d'une impédance à deux dimensions d'espace est un peu plus complexe et ne figure pas ici. Le calcul des estimations d'énergie pour ces deux sous-problèmes repose en grande partie sur la preuve proposée dans [Petit-Bergez, 2006]. L'idée directrice est de définir un système d'équations étendu faisant intervenir les variables initiales et leurs dérivées premières , [Métral, Vacus, 1999], puis de montrer que ce système est fortement bien posé et de calculer les estimations correspondantes. Les résultats obtenus sont maintenant exposés.

Théorème 1 : Problème aux limites pour le demi-espace. Soit $\Omega$ le demi-espace $\left[z_{1},+\infty\right] \times \mathbb{R}$. Soit $h(x, t) \in H^{7 / 2,7 / 2}(\mathbb{R} \times[0, T])$ telle que $h(x, 0)=0, \partial_{t} h(x, 0)=0, \partial_{t t} h(x, 0)=0$. Pour $\left(\sigma_{x}, \sigma_{z}\right) \in\left(W^{1, \infty}(\Omega)\right)^{2}$, $c \in C^{\infty}(\Omega), \alpha \in C^{\infty}(\Omega)$ tels que

$$
0<c_{\min } \leq c(x, z) \leq c_{\max }, \quad 0<\alpha_{\min } \leq \alpha(x, z) \leq \alpha_{\max }, \quad \text { alors }
$$

$\exists\left(C_{1}, C_{2}\right) \in \mathbb{R}^{2}$, tels que pour toute solution suffisamment régulière de (5), (6) on aie $\left\|\left(u_{x}, u_{z}, p_{x}+p_{z}\right)\right\|_{L^{\infty}\left(0, T ; H^{1}(\Omega)\right)}+\left\|\left(\sigma_{x} u_{x}, \sigma_{z} u_{z}, c\left(\sigma_{x} p_{x}+\sigma_{z} p_{z}\right)\right)\right\|_{L^{\infty}\left(0, T ; L^{2}(\Omega)\right)} \leq$

$\frac{N}{M} e^{\frac{(\gamma+1) T}{2 M^{2}}}\left\|\left(u_{x}, u_{z}, p_{x}+p_{z}\right)(., ., 0)\right\|_{H^{1}(\Omega)}+\frac{N}{M} e^{\frac{(\gamma+1) T}{2 M^{2}}}\left\|\left(\sigma_{x} u_{x}, \sigma_{z} u_{z}, c\left(\sigma_{x} p_{x}+\sigma_{z} p_{z}\right)\right)(., ., 0)\right\|_{L^{2}(\Omega)}$

$$
\begin{gathered}
+\frac{C_{1} N}{M} e^{\frac{(\gamma+1) T}{2 M^{2}}}\|h\|_{H^{7 / 2,7 / 2}([0, T] \times \mathbb{R})}+C_{2}\|h\|_{H^{7 / 2,7 / 2}([0, T] \times \mathbb{R})} \quad \text { avec } \\
\gamma(\alpha, c)=\sup _{x, z}\left\|\partial_{x}(\tilde{H} \tilde{A})+\partial_{z}(\tilde{H} \tilde{B})+2 \tilde{H} \tilde{S}\right\|_{2} \\
M(\alpha, c)=\min \min _{x, z}\left\{\alpha(x, z) c(x, z), \frac{c(x, z)}{\alpha(x, z)}, \frac{4 \alpha^{2}+c^{2}-\sqrt{16 \alpha^{4}+c^{4}}}{2 \alpha c}(x, z)\right\}, \\
N(\alpha, c)=\max \left\{\|\alpha c\|_{\infty},\left\|\frac{c}{\alpha}\right\|_{\infty},\left\|\frac{4 \alpha^{2}+c^{2}+\sqrt{16 \alpha^{4}+c^{4}}}{2 \alpha c}\right\|_{\infty}\right\},
\end{gathered}
$$

les matrices $\widetilde{H}, \widetilde{A}, \widetilde{B}, \widetilde{S}$ étant définies par la suite.

Théorème 2 : Problème pour deux sous domaines séparés par une interface. Soient $\Omega_{1}=\mathbb{R} \times$ $\mathbb{R}_{-}, \quad \Omega_{2}=\mathbb{R} \times \mathbb{R}_{+}, \quad \Omega=\Omega_{1} \cup \Omega_{2}$, et $\left(\sigma_{x}, \sigma_{z}\right) \in\left(W^{1, \infty}(\Omega)\right)^{2},\left(u_{x}, u_{z}, p_{x}, p_{z}\right) \in\left(H^{1}(\Omega)\right)^{4}, c_{1} \in C^{\infty}\left(\Omega_{1}\right)$, 
$c_{2} \in C^{\infty}\left(\Omega_{2}\right), \alpha_{1} \in C^{\infty}\left(\mathbb{R}_{-}\right), \alpha_{2} \in C^{\infty}\left(\mathbb{R}_{+}\right)$, tels que

$$
\begin{gathered}
0<c_{\min } \leq c_{i}(x, z) \leq c_{\max }, \quad 0<\alpha_{\min } \leq \alpha_{i}(z) \leq \alpha_{\max }, \quad i=1,2, \\
\text { alors, pour toute solution suffisamment régulière de }(5) \text { dans } \mathbb{R}^{2}, \\
\left\|\left(u_{x}, u_{z}, p_{x}+p_{z}\right)(., ., t)\right\|_{H^{1}(\Omega)}^{2}+\left\|\left(\sigma_{x} u_{x}, \sigma_{z} u_{x}, c\left(\sigma_{x} p_{x}+\sigma_{z} p_{z}\right)\right)(., ., t)\right\|_{L^{2}(\Omega)}^{2} \leq \\
\left(\frac{N^{\prime}}{M^{\prime}}\right)^{2} e^{\frac{\gamma^{\prime} t}{\left(M^{\prime}\right)^{2}}}\left(\left\|\left(u_{x}, u_{z}, p_{x}+p_{z}\right)(., ., 0)\right\|_{H^{1}(\Omega)}^{2}\left\|+\left(\sigma_{x} u_{x}, \sigma_{z} u_{z}, c\left(\sigma_{x} p_{x}+\sigma_{z} p_{z}\right)\right)(., ., 0)\right\|_{L^{2}(\Omega)}^{2}\right), \\
\text { avec } \gamma^{\prime} \leq \max _{i=1,2} \gamma\left(\alpha_{i}, c_{i}\right), \quad M^{\prime}=\min _{i=1,2} M\left(\alpha_{i}, c_{i}\right), \quad N^{\prime}=\max _{i=1,2} N\left(\alpha_{i}, c_{i}\right) .
\end{gathered}
$$

\subsection{Démonstrations des théorèmes}

\subsubsection{Définition du système étendu}

Soit

$$
U_{1}(x, z, t)=\left[\begin{array}{c}
u_{x} \\
u_{z} \\
p
\end{array}\right], \quad U_{2}(x, z, t)=\partial_{x} U_{1}, \quad U_{3}(x, z, t)=\partial_{z} U_{1}, \quad U_{4}(x, z, t)=\left[\begin{array}{c}
\sigma_{x} u_{x} \\
\sigma_{z} u_{z} \\
c\left(\sigma_{x} p_{x}+\sigma_{z} p_{z}\right)
\end{array}\right]
$$

Le système étendu est défini par les équations

$$
\left\{\begin{array}{c}
\partial_{t} U_{1}-A(x, z) \partial_{x} U_{1}-B(x, z) \partial_{z} U_{1}+C(x, z) U_{1}+E U_{4}=0 \\
\partial_{t} U_{2}-A(x, z) \partial_{x} U_{2}-B(x, z) \partial_{z} U_{2}+C(x, z) U_{2}-\partial_{x} A(x, z) U_{2}-\partial_{x} B(x, z) U_{3}+\partial_{x} C(x, z) U_{1}+E \partial_{x} U_{4}=0 \\
\partial_{t} U_{3}-A(x, z) \partial_{x} U_{3}-B(x, z) \partial_{z} U_{3}+C(x, z) U_{3}-\partial_{z} A(x, z) U_{2}-\partial_{z} B(x, z) U_{3}+\partial_{z} C(x, z) U_{1}+E \partial_{z} U_{4}=0 \\
\partial_{t} U_{4}-S^{1}(x, z) U_{1}-S^{2}(x, z) U_{2}-S^{3}(x, z) U_{3}+S^{4}(x, z) U_{4}=0 \\
\text { où } A(x, z)=\left(\begin{array}{ccc}
0 & 0 & \alpha c \\
0 & 0 & 0 \\
\frac{c}{\alpha} & 0 & 0
\end{array}\right), \quad B(x, z)=\left(\begin{array}{ccc}
0 & 0 & 0 \\
0 & 0 & \alpha c \\
0 & \frac{c}{\alpha} & 0
\end{array}\right)
\end{array}\right.
$$

sont les matrices associées au système des équations d'Euler (1) et $C, E, S^{i}, i=1, \ldots, 4$ sont définies par

$$
\begin{gathered}
C(x, z)=\left(\begin{array}{ccc}
c \sigma_{x} & 0 & 0 \\
0 & c \sigma_{z} & 0 \\
0 & 0 & 0
\end{array}\right), \quad E=\left(\begin{array}{ccc}
0 & 0 & 0 \\
0 & 0 & 0 \\
0 & 0 & 1
\end{array}\right), \\
S^{1}(x, z)=\left(\begin{array}{ccc}
0 & 0 & 0 \\
0 & 0 & 0 \\
0 & 0 & c^{2} \sigma_{x} \sigma_{z}
\end{array}\right), \quad S^{2}(x, z)=\left(\begin{array}{ccc}
0 & 0 & \sigma_{x} \alpha c \\
0 & 0 & 0 \\
c^{2} & 0 & 0 \\
\sigma_{x} \frac{\alpha}{\alpha} & 0
\end{array}\right), \\
S^{3}(x, z)=\left(\begin{array}{ccc}
0 & 0 & 0 \\
0 & 0 & \sigma_{z} \alpha c \\
0 & \sigma_{z} \frac{c^{2}}{\alpha} & 0
\end{array}\right), \quad S^{4}(x, z)=\left(\begin{array}{ccc}
c \sigma_{x} 0 & 0 & 0 \\
0 & c \sigma_{z} & 0 \\
0 & 0 & c\left(\sigma_{x}+\sigma_{z}\right)
\end{array}\right) .
\end{gathered}
$$


Ainsi en posant

$$
\begin{gathered}
\widetilde{U}(x, z, t)=\left[\begin{array}{c}
U_{1} \\
U_{2} \\
\\
U_{3} \\
U_{4}
\end{array}\right], \quad \widetilde{A}(x, z)=\left(\begin{array}{cccc}
A & 0 & 0 & 0 \\
0 & A & 0 & -E \\
0 & 0 & A & 0 \\
0 & 0 & 0 & 0
\end{array}\right), \quad \widetilde{B}(x, z)=\left(\begin{array}{cccc}
B & 0 & 0 & 0 \\
0 & B & 0 & 0 \\
0 & 0 & B & -E \\
0 & 0 & 0 & 0
\end{array}\right), \\
\widetilde{S}(x, z)=\left(\begin{array}{ccccc}
C & 0 & 0 & E \\
\partial_{x} C & C-\partial_{x} A & -\partial_{x} B & 0 \\
\partial_{z} C & -\partial_{z} A & C-\partial_{z} B & 0 \\
-S^{1} & -S^{2} & -S^{3} & S^{4}
\end{array}\right),
\end{gathered}
$$

il est possible d'écrire le système étendu sous la forme condensée

$$
\partial_{t} \widetilde{U}-\widetilde{A} \partial_{x} \widetilde{U}-\widetilde{B} \partial_{z} \widetilde{U}+\widetilde{S} \widetilde{U}=0 .
$$

\subsubsection{Existence d'un symétriseur défini positif pour le problème étendu}

Définition 1 Soit un opérateur

$$
\begin{gathered}
\mathcal{P}\left(x, t, \partial_{x}\right)=\sum_{j=1}^{J} A_{j}(x, t) \partial_{x_{j}}, \\
\text { de symbole } \quad P(x, t, i \omega)=i \sum_{j=1}^{J} \omega_{j} A_{j}(x, t) \quad \omega \in \mathbb{R}^{J} .
\end{gathered}
$$

Un symétriseur de cet opérateur est une matrice hermitienne $H(x, t, \omega)$ définie positive pour tout $x \in \mathbb{R}^{J}$, $t \in[0, T], \omega \in \mathbb{R}^{J},|w|=1, C^{\infty}$ par rapport à tous ses arguments, et vérifiant

$$
H(x, t, \omega) P(x, t, \omega)+P^{*}(x, t, \omega) H(x, t, \omega)=0 .
$$

Lemme 1. Il existe un symétriseur réel $\widetilde{H}(x, z)$ pour l'opérateur $\mathcal{P}_{1}=\widetilde{A} \partial_{x}+\widetilde{B} \partial_{z}$. De plus, son indépendance par rapport aux variables $\omega_{1}$ et $\omega_{2}$ lui confère la propriété de symétriser simultanément les matrices $\widetilde{A}$ et $\widetilde{B}$.

Démonstration. Un symétriseur $H(x, z)$ pour l'équation d'Euler (1) est

On vérifie que

$$
H(x, z)=\left(\begin{array}{ccc}
\frac{c}{\alpha} & 0 & 0 \\
0 & \frac{c}{\alpha} & 0 \\
0 & 0 & \alpha c
\end{array}\right) .
$$

$$
\widetilde{H}(x, z)=\left(\begin{array}{cccc}
H & 0 & 0 & 0 \\
0 & H & 0 & X_{1} \\
0 & 0 & H & X_{2} \\
0 & X_{1}^{T} & X_{2}^{T} & L
\end{array}\right)
$$


est un symétriseur de $\mathcal{P}_{1}$ qui symétrise simultanément $\widetilde{A}$ et $\widetilde{B}$, avec

$$
X_{1}=\left(\begin{array}{ccc}
0 & 0 & -1 \\
0 & 0 & 0 \\
0 & 0 & 0
\end{array}\right), \quad X_{2}=\left(\begin{array}{ccc}
0 & 0 & 0 \\
0 & 0 & -1 \\
0 & 0 & 0
\end{array}\right), \quad L(x, z)=\left(\begin{array}{ccc}
1 & 0 & 0 \\
0 & 1 & 0 \\
0 & 0 & 4 \frac{\alpha}{c}
\end{array}\right)
$$

Remarque. L'existence du symétriseur $\widetilde{H}(x, z)$ prouve, d'après [Kreiss, Lorenz, 2004], que le problème de Cauchy pour le système étendu et des coefficients $\alpha(x, z)$ et $c(x, z) C^{\infty}$ est fortement hyperbolique et donc fortement bien posé. Toutefois, le cas qui nous intéresse est celui d'un domaine borné avec d'éventuelles discontinuités d'impédance $\alpha(x, z)$, il est donc nécessaire de calculer directement les estimations.

2.2.3. Estimations pour le demi-espace $z>0$

Soit $\Omega=\left[z_{1},+\infty\right] \times \mathbb{R}^{+}$. L'impédance $\alpha(x, z)$ et la vitesse $c(x, z)$ vérifient

$$
\alpha(x, z) \in C^{\infty}\left(\Omega ; R_{+}^{*}\right), \quad c(x, z) \in C^{\infty}\left(\Omega ; R_{+}^{*}\right) .
$$

Le symétriseur $\widetilde{H}(x, z)$ induit le produit scalaire

$$
(\widetilde{U}, \widetilde{V})_{\widetilde{H}}=(\widetilde{U}, \widetilde{H} \widetilde{V})
$$

La norme induite par ce produit scalaire est équivalente à la norme $L^{2}$, et $M\|\widetilde{U}\|_{L^{2}} \leq\|\widetilde{U}\|_{\widetilde{H}} \leq N\|\widetilde{U}\|_{L^{2}}$, où $M$ et $N$ sont définis par l'équation (9).

Lemme 2. Pour $h \in H^{7 / 2,7 / 2}([0, T] \times \mathbb{R})$ telle que $h(x, 0)=0, \quad \partial_{t} h(x, 0)=0, \quad \partial_{t t} h(x, 0)=0, \quad \exists\left(C_{1}, C_{2}\right) \in$ $\mathbb{R}^{2}$ tels que pour $\widetilde{U}$ solution de (14),

$$
\|\widetilde{U}(t)\|_{L^{\infty}\left(0, T ; L^{2}(\Omega)\right)} \leq \frac{1}{M} \exp ^{\frac{(\gamma+1) T}{2 M^{2}}}\left(N\|\widetilde{U}(0)\|_{L^{2}(\Omega)}++C_{1}\|h\|_{H^{7 / 2,7 / 2}([0, T] \times \mathbb{R})}\right)+C_{2}\|h\|_{H^{7 / 2,7 / 2}([0, T] \times \mathbb{R})} .
$$

où $\gamma$ est défini par l'équation (9).

Démonstration. La régularité de $h$ permet de définir un système équivalent au système (14), avec des conditions initiales et des conditions au bord homogènes, et un second membre non nul. Ce système équivalent est obtenu par la définition de relèvements appropriés. Notant $\bar{u}_{x}, \bar{u}_{z}, \bar{p}, \bar{v}, U_{x}, U_{z}, P, V$ tels que

$$
\begin{gathered}
\bar{u}_{x}=u_{x}-U_{x}, \quad \bar{u}_{z}=u_{z}-U_{z}, \quad \bar{p}=p-P, \quad \bar{v}=v-V, \\
\bar{U}=\left(\bar{u}_{x}, \bar{u}_{z}, \bar{p}, \partial_{x} \bar{u}_{x}, \partial_{x} \bar{u}_{z}, \partial_{x} \bar{p}, \partial_{z} \bar{u}_{x}, \partial_{z} \bar{u}_{z}, \partial_{z} \bar{p}, \sigma_{x} \bar{u}_{x}, \sigma_{z} \bar{u}_{z}, \bar{v}\right),
\end{gathered}
$$

$\bar{U}$ est solution de

$$
\partial_{t} \bar{U}-\widetilde{A} \partial_{x} \bar{U}-\widetilde{B} \partial_{z} \bar{U}-\widetilde{S} \bar{U}=F(x, z, t)
$$

avec

$$
\left\{\begin{array}{l}
\exists C_{1} \in \mathbb{R}, \quad\|F\|_{L^{2}\left(0, T ; L^{2}(\Omega)\right)} \leq C_{1}\|h\|_{H^{7 / 2,7 / 2}([0, T] \times \mathbb{R})}, \\
\forall(x, z) \in \Omega, \quad \widetilde{U}(x, z, 0)=\bar{U}(x, z, 0)=0, \\
\forall(x, t) \in \mathbb{R} \times[0, T], \quad \bar{p}(x, 0, t)=0, \quad \partial_{z} \bar{u}_{z}(x, 0, t)=0, \quad v(x, 0, t)=0 .
\end{array}\right.
$$


Soient $\quad \check{A}=\widetilde{H} \widetilde{A}, \quad \check{B}=\widetilde{H} \widetilde{B}, \check{S}=\widetilde{H} \widetilde{S}$, alors,$\quad \frac{d}{d t}\|\bar{U}(t)\|_{\widetilde{H}}^{2}=\left(\bar{U}, \widetilde{H} \frac{d \bar{U}}{d t}\right)+\left(\widetilde{H} \bar{U}, \frac{d \bar{U}}{d t}\right)$

$$
=\int_{\Omega}\left(\bar{U}(x, z, t), \check{A} \partial_{x} \bar{U}(x, z, t)\right)+\left(\bar{U}(x, z, t), \check{B} \partial_{z} \bar{U}(x, z, t)\right) d x d z
$$

$+\int_{\Omega}\left(\check{A} \bar{U}(x, z, t), \partial_{x} \bar{U}(x, z, t)\right)+\left(\check{B} \bar{U}(x, z, t), \partial_{z} \bar{U}(x, z, t)\right)-2(\bar{U}(x, z, t), \check{S} \bar{U}(x, z, t))+2(F(x, z, t), \bar{U}(x, z, t)) d x d z$.

Par intégration par parties des troisième et quatrième termes de la dernière ligne, il vient

$$
\begin{gathered}
\frac{d}{d t}\|\bar{U}(t)\|_{\widetilde{H}}^{2}=-\int_{\Omega}\left(\bar{U}(x, z, t), 2 \check{S} \bar{U}(x, z, t)+\partial_{x}(\check{A}) \bar{U}(x, z, t)+\partial_{z}(\check{B}) \bar{U}(x, z, t)\right) \\
-2(F(x, z, t), \bar{U}(x, z, t)) d x d z-\int_{\mathbb{R}}(\check{B} \bar{U}(x, 0, t), \bar{U}(x, 0, t)) d x \\
\text { Or } \quad \int_{\mathbb{R}}(\check{B} \bar{U}(x, 0, t), \bar{U}(x, 0, t)) d x=2 \int_{\mathbb{R}} c^{2}(x, 0)\left(\bar{u}_{z}(x, 0, t) \bar{p}(x, 0, t)+\partial_{x} u_{z}(x, 0, t) \partial_{x} \bar{p}(x, 0, t)\right) \\
+\alpha(x, 0) c(x, 0) \partial_{z} \bar{p}(x, 0, t)\left(\frac{c(x, 0)}{\alpha(x, 0)} \partial_{z} \bar{u}_{z}(x, 0, t)-\bar{v}(x, 0, t)\right) d x
\end{gathered}
$$

Cependant, $\bar{p}(x, 0, t)=0$, d'où $\partial_{x} \bar{p}(x, 0, t)=0$. D'autre part, $\partial_{z} \bar{u}_{z}(x, 0, t)=0$ et $\bar{v}(x, 0, t)=0$. Le terme de bord apparaissant dans les estimations d'énergie est donc nul et

$$
\frac{d}{d t}\|\bar{U}(t)\|_{\widetilde{H}}^{2} \leq\left(\sup _{x, z}\left\|\partial_{x} \check{A}+\partial_{z} \check{B}+2 \check{S}\right\|_{2}+1\right)\|\bar{U}(t)\|_{L^{2}(\Omega)}^{2}+\|\tilde{F}(t)\|_{L^{2}(\Omega)}^{2} .
$$

D'où

$$
\frac{d}{d t}\|\bar{U}(t)\|_{\widetilde{H}}^{2} \leq \frac{\gamma+1}{M^{2}}\|\bar{U}(t)\|_{\widetilde{H}}^{2}+\|\tilde{F}(t)\|_{L^{2}(\Omega)}^{2} .
$$

L'utilisation du lemme de Gronwall et les inégalités relatives au produit scalaire induit par $\widetilde{H}$ permettent d'établir

$$
\|\bar{U}(t)\|_{L^{2}(\Omega)}^{2} \leq \frac{1}{M^{2}} e^{\frac{(\gamma+1) t}{M^{2}}}\left(N^{2}\|\bar{U}(0)\|_{L^{2}(\Omega)}^{2}+\int_{0}^{t}\|\tilde{F}(\tau)\|_{L^{2}(\Omega)}^{2} d \tau\right) .
$$

Il vient d'après ce qui a été établi au début de la démonstration

$$
\|\bar{U}(t)\|_{L^{\infty}\left(0, T ; L^{2}(\Omega)\right)} \leq \frac{1}{M} \exp ^{\frac{(\gamma+1) T}{2 M^{2}}}\left(N\|\widetilde{U}(0)\|_{L^{2}(\Omega)}+C_{1}\|h\|_{H^{7 / 2,7 / 2}([0, T] \times \mathbb{R})}\right) .
$$

Or, par les propriétés des relèvements, $\exists C_{2} \in \mathbb{R}, \quad\|\bar{U}-\widetilde{U}\|_{L^{\infty}\left(0, T ; L^{2}(\Omega)\right)} \leq C_{2}\|h\|_{H^{7 / 2,7 / 2}([0, T] \times \mathbb{R})}$, et par l'inégalité triangulaire on déduit

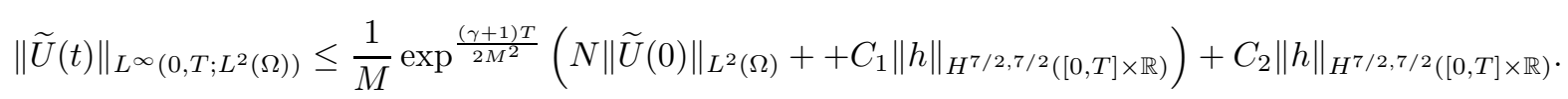




\subsubsection{Estimations pour le problème avec interface}

Soient maintenant deux demi-espaces $\Omega_{1}, \Omega_{2}$ séparés par une interface située en $z=0$.

$$
\Omega_{1}=\mathbb{R} \times \mathbb{R}_{-}, \quad \Omega_{2}=\mathbb{R} \times \mathbb{R}_{+} .
$$

L'espace entier est noté $\Omega=\Omega_{1} \cup \Omega_{2}$. Nous nous plaçons dans le cas d'un milieu de propagation stratifié. Chacun de ces sous-domaines est caractérisé par une impédance $\alpha_{i}$ et une vitesse $c_{i}$, pour $i=1,2$, telles que

$$
\alpha_{1}(z) \in C^{\infty}\left(\mathbb{R}_{-} ; \mathbb{R}_{+}^{*}\right), \quad \alpha_{2}(z) \in C^{\infty}\left(\mathbb{R}_{+} ; \mathbb{R}_{+}^{*}\right), \quad c_{i}(x, z) \in C^{\infty}\left(\Omega_{i} ; \mathbb{R}_{+}^{*}\right), \quad i=1,2
$$

La définition des symétriseurs $\widetilde{H}^{i}$ sur les domaines $\Omega^{i}$ induit des produits scalaires qui sont notés $(.,.) \widetilde{H}^{i}$. Les normes associées à ces produits scalaires sont équivalentes aux normes $L^{2}$ définies sur ces domaines, et induisent les constantes $M_{i}$ et $N_{i}$ définies par (9).

Lemme 3. Pour $\widetilde{U}(x, z, t)$ solution de (14) sur le domaine $\Omega$,

avec $\gamma^{\prime}, M^{\prime}$ et $N^{\prime}$ définis par (12).

$$
\|\widetilde{U}(t)\|_{L^{2}}^{2} \leq \frac{N^{\prime 2}}{M^{\prime 2}} e^{\frac{\gamma^{\prime} t}{M^{\prime 2}}}\|\widetilde{U}(0)\|_{L^{2}}^{2}
$$

Démonstration. Soit $\widetilde{U}^{i}$ la restriction à $\Omega_{i}$ de la solution $\widetilde{U}$ du système (14) dans le domaine $\Omega$. Les quantités continues à l'interface sont la pression $p$ et la vitesse normale à l'interface $u_{z}$. D'autre part, la vitesse du son $c(x, z)$ est elle-même continue. De ceci se déduit la continuité à l'interface des termes

$$
c^{2}(x, 0) u_{z}(x, 0, t) p(x, 0, t) \quad \text { et } c^{2}(x, 0) \partial_{x} u_{z}(x, 0, t) \partial_{x} p(x, 0, t)
$$

D'autre part, d'après (5),

$$
\begin{aligned}
& \partial_{t} \frac{u_{x}^{1}\left(x, 0^{-}, t\right)}{\alpha^{1}\left(0^{-}\right)}+\frac{\sigma_{x}(x)}{\alpha^{1}\left(0^{-}\right)} u_{x}^{1}\left(x, 0^{-}, t\right)=c^{1}\left(x, 0^{-}\right) \partial_{x} p^{1}\left(x, 0^{-}, t\right) \\
& =c^{2}\left(x, 0^{+}\right) \partial_{x} p^{2}\left(x, 0^{+}, t\right)=\partial_{t} \frac{u_{x}^{2}\left(x, 0^{+}, t\right)}{\alpha^{2}\left(0^{+}\right)}+\frac{\sigma_{x}(x)}{\alpha^{2}\left(0^{+}\right)} u_{x}^{2}\left(x, 0^{+}, t\right)
\end{aligned}
$$

Comme $u_{x}^{i}(x, 0,0)=0$ pour $i=1,2$,

$$
\frac{u_{x}^{1}\left(x, 0^{-}, t\right)}{\alpha\left(0^{-}\right)}=\frac{u_{x}^{2}\left(x, 0^{+}, t\right)}{\alpha\left(0^{+}\right)}
$$

La continuité de $p$ à l'interface implique également celle de $\partial_{t} p$, qui implique, en utilisant une nouvelle fois $(5)$,

$$
\begin{gathered}
\frac{c^{1}\left(x, 0^{-}\right)}{\alpha\left(0^{-}\right)} \partial_{x} u_{x}^{1}\left(x, 0^{-}, t\right)+\frac{c^{1}\left(x, 0^{-}\right)}{\alpha^{1}\left(0^{-}\right)} \partial_{z} u_{z}^{1}\left(x, 0^{-}, t\right)-v^{1}\left(x, 0^{-}, t\right)= \\
\frac{c^{2}\left(x, 0^{+}\right)}{\alpha\left(0^{+}\right)} \partial_{x} u_{x}^{2}\left(x, 0^{+}, t\right)+\frac{c^{2}\left(x, 0^{+}\right)}{\alpha^{2}\left(0^{+}\right)} \partial_{z} u_{z}^{2}\left(x, 0^{+}, t\right)-v^{2}\left(x, 0^{+}, t\right)
\end{gathered}
$$

En dérivant la relation (18) suivant $x, \alpha$ ne dépendant pas de $x$, il vient

$$
\frac{c^{1}\left(x, 0^{-}\right)}{\alpha^{1}\left(0^{-}\right)} \partial_{z} u_{z}^{1}\left(x, 0^{-}, t\right)-v^{1}\left(x, 0^{-}, t\right)=\frac{c^{2}\left(x, 0^{+}\right)}{\alpha^{2}\left(0^{+}\right)} \partial_{z} u_{z}^{2}\left(x, 0^{+}, t\right)-v^{2}\left(x, 0^{+}, t\right)
$$


Ces relations à l'interface permettent d'aborder le problème d'estimation d'énergie. Soient

$$
\begin{gathered}
\check{A}_{i}=\widetilde{H}_{i} \widetilde{A}_{i}, \quad \check{B}_{i}=\widetilde{H}_{i} \widetilde{B}_{i}, \quad \check{S}_{i}=\widetilde{H}_{i} \widetilde{S}_{i}, \quad i=1,2 . \\
\text { Alors } \frac{d}{d t}\left\|\widetilde{U}^{1}(t)\right\|_{\widetilde{H}_{1}}^{2}+\frac{d}{d t}\left\|\widetilde{U}^{2}(t)\right\|_{\widetilde{H}_{2}}^{2}= \\
-\int_{\Omega_{1}}\left(\widetilde{U}^{1}(x, z, t), \partial_{x}\left(\check{A}^{1}\right) \widetilde{U}^{1}(x, z, t)+\partial_{z}\left(\check{B}^{1}\right) \widetilde{U}^{1}(x, z, t)+2 \check{S}^{1} \widetilde{U}^{1}(x, z, t)\right) d x d z \\
-\int_{\Omega_{2}}\left(\widetilde{U}^{2}(x, z, t), \partial_{x}\left(\check{A}^{2}\right) \widetilde{U}^{2}(x, z, t)+\partial_{z}\left(\check{B}^{2}\right) \widetilde{U}^{2}(x, z, t)+2 \check{S}^{2} \widetilde{U}^{2}(x, z, t)\right) d x d z \\
+\int_{\mathbb{R}}\left(\check{B}^{1} \widetilde{U}^{1}(x, 0, t), \widetilde{U}^{1}(x, 0, t)\right)-\left(\check{B}^{2} \widetilde{U}^{2}(x, 0, t), \widetilde{U}^{2}(x, 0, t)\right) d x . \\
\operatorname{Comme~précédemment~}, \quad \int_{\mathbb{R}}\left(\check{B}^{i} \widetilde{U}^{i}(x, 0, t), \widetilde{U}^{i}(x, 0, t)\right)= \\
2 \int_{\mathbb{R}} c_{i}^{2}(x, 0)\left(u_{z}^{i}(x, 0, t) p^{i}(x, 0, t)+\partial_{x} u_{z}^{i}(x, 0, t) \partial_{x} p^{i}(x, 0, t)\right) d x \\
+2 \int_{\mathbb{R}} c_{i}^{2}(x, 0) \alpha^{i}(0) c^{i}(x, 0) \partial_{z} p^{i}(x, 0, t)\left(\frac{c^{i}(x, 0)}{\alpha^{i}(0)} \partial_{z} u_{z}^{i}(x, 0, t)-v^{i}(x, 0, t)\right) d x .
\end{gathered}
$$

D'après les relations à l'interface établies plus haut, il ressort que ces termes de bord s'annulent entre eux. Il vient alors

$$
\begin{gathered}
\frac{d}{d t}\left\|\widetilde{U}^{1}(t)\right\|_{\widetilde{H}_{1}}^{2}+\frac{d}{d t}\left\|\widetilde{U}^{2}(t)\right\|_{\widetilde{H}_{2}}^{2}= \\
-\int_{\Omega_{1}}\left(\widetilde{U}^{1}(x, z, t), \partial_{x}\left(\check{A}^{1}\right) \widetilde{U}^{1}(x, z, t)+\partial_{z}\left(\check{B}^{1}\right) \widetilde{U}^{1}(x, z, t)+2 \breve{S}^{1} \widetilde{U}^{1}(x, z, t)\right) d x d z \\
-\int_{\Omega_{2}}\left(\widetilde{U}^{2}(x, z, t), \partial_{x}\left(\check{A}^{2}\right) \widetilde{U}^{2}(x, z, t)-\partial_{z}\left(\check{B}^{2}\right) \widetilde{U}^{2}(x, z, t)+2 \check{S}^{2} \widetilde{U}^{2}(x, z, t)\right) d x d z .
\end{gathered}
$$

d'où, par l'inégalité de Cauchy-Schwarz,

$$
\frac{d}{d t}\left(\left\|\widetilde{U}^{1}(t)\right\|_{\widetilde{H}_{1}}^{2}+\left\|\widetilde{U}^{2}(t)\right\|_{\widetilde{H}_{2}}^{2}\right) \leq \frac{\gamma^{\prime}}{\left(M^{\prime}\right)^{2}}\left(\left\|\widetilde{U}^{1}(t)\right\|_{\widetilde{H}_{1}}^{2}+\left\|\widetilde{U}^{2}(t)\right\|_{\widetilde{H}_{2}}^{2}\right) .
$$

L'utilisation du lemme de Gronwall et les inégalités inhérentes aux produits scalaires induits par les symétriseurs $\widetilde{H}_{i}$ sur les sous-espaces $\Omega_{i}$ permettent de conclure.

Ces résultats montrent donc le caractère faiblement bien posé des équations PML de Bérenger, appliquées aux équations d'Euler 2D linéarisées autour d'une vitesse nulle, dans un milieu borné, héterogène, et discontinu suivant $z$. L'extension de ce résultat au problème $3 \mathrm{D}$ fait actuellement l'objet de recherches. Un symétriseur associé au probléme 3D a été calculé, ce qui indique que le résultat tient pour le problème de Cauchy à coefficients variables $C^{\infty}$. Cependant, il reste à prolonger l'étude au problème en milieu borné discontinu 3D. 


\section{RÉSUltats NUMÉRiQUeS}

\subsection{Description de l'expérience}

Des tests numériques sont réalisés pour valider l'efficacité de cette formulation PML. Le domaine dans lequel la propagation des ondes acoustiques est étudiée est rectangulaire. Les simulations sont effectuées pour des modèles de vitesse et d'impédance de complexité croissante (milieu homogène, milieu bi-couche, milieu très hétérogène issu de données réelles (figure 1)), afin de mettre en évidence les difficultés issues de l'hétérogénéité du milieu. A chaque fois, deux cas sont considérés. Le premier correspond à l'expérience témoin : les modèles initiaux de vitesse et d'impédance sont prolongés par continuité dans les directions $x$ et $z>0$ de manière à définir un modèle "demi-espace" englobant le domaine dans lequel la propagation est étudiée. Les dimensions de ce modèle "demi-espace" sont choisies de manière à ce que le signal émis par la source ne subisse pas de réflexion totale sur les extrémités du domaine pendant le temps de propagation défini au préalable $T=2 s$. Le deuxième correspond à l'expérience PML : le modèle initial est à nouveau prolongé par continuité dans les directions $x$ et $z>0$ mais pour 20 points de discrétisation seulement. Ceci permet de définir les couches PML dans lesquelles un terme d'absorption non nul est introduit. Une comparaison entre les champs de vitesse verticale est alors effectuée pour un temps donné afin de juger de l'efficacité des PML.
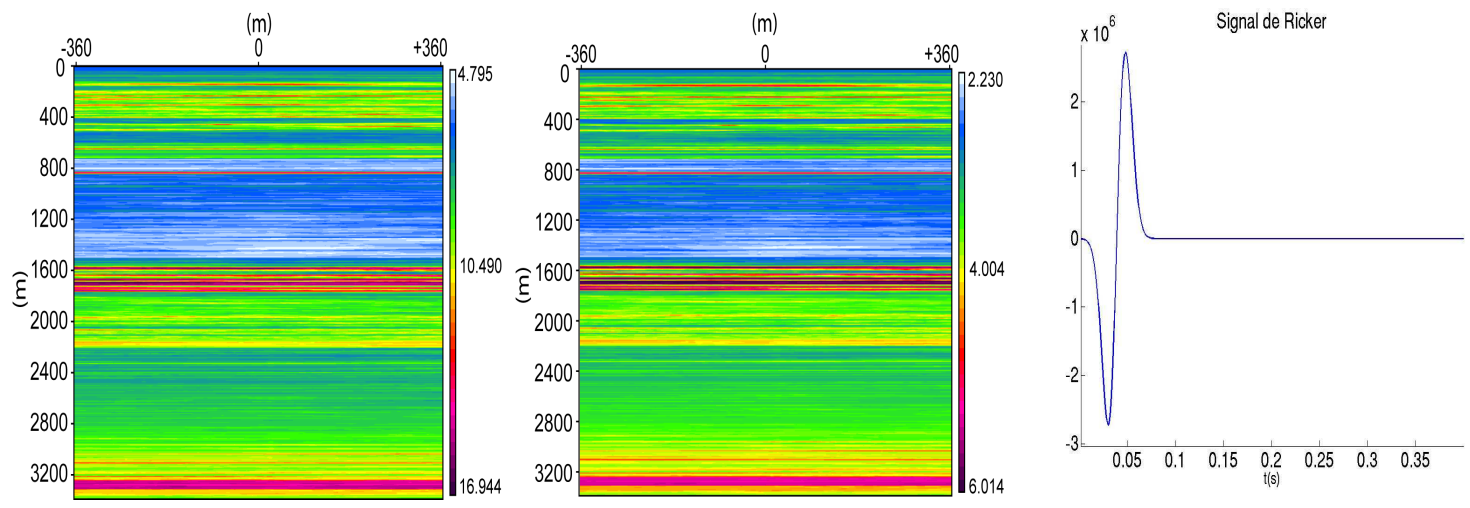

FiguRE 1. Modèles d'impédance (gauche) et de vitesse de propagation (centre), signal de ricker (droite)

\subsection{Paramètres de l'expérience}

Le signal $s(t)=5 \times 10^{8}\left(t-\frac{1}{f}\right) \exp \left(-\Pi^{2} f^{2}\left(t-\frac{1}{f}\right)^{2}\right)$ émis par la source est un signal typique utilisé en géophysique, appelé signal de Ricker (figure 1). C'est un signal à moyenne nulle centré sur une fréquence $f=25 \mathrm{~Hz}$. La source $\widetilde{s}(x, z, t)$ utilisée est centrée en $\left(x_{S}=0, z_{S}=1700\right)$ et telle que

$$
\widetilde{s}(x, z, t)=s(t) \exp \left(-\frac{\left(x-x_{S}\right)^{2}}{10^{4}}\right) \exp \left(-\frac{\left(z-z_{S}\right)^{2}}{10^{4}}\right)
$$

ce qui garantit une bonne régularité spatiale et temporelle. Les coefficients d'absorption utilisés pour le modèle PML sont choisis conformément aux expériences numériques de [Hu, 1996],

$$
\begin{gathered}
\sigma_{x}(x)=\sigma_{0, x}\left(\frac{d_{x}(x)}{D_{x}}\right)^{n}, \quad \sigma_{z}(z)=\sigma_{0, z}\left(\frac{d_{z}(z)}{D_{z}}\right)^{n} \\
\text { avec } \quad \sigma_{0, x}=8(n+1) / D_{x}, \quad \sigma_{0, z}=8(n+1) / D_{z}, \quad n=3 .
\end{gathered}
$$

$D_{x}$ et $D_{z}$ représentent l'épaisseur des PML respectivement suivant $x$ et $z$, et les fonctions $d_{x}(x)$ et $d_{z}(z)$ mesurent la distance séparant le point courant du début de la couche PML, repsectivement suivant $x$ et $z$.

Le domaine initial considéré est $\Omega=[-360,360] \times[0,3400]$. Pour une bonne représentation de l'onde, il faut 
vérifier la condition suivante, $\lambda$ désignant la longueur d'onde caractéristique :

$$
\Delta x \leq \frac{\lambda}{10}, \quad \Delta z \leq \frac{\lambda}{10}, \quad \text { soit } \Delta x \leq 14 m, \quad \Delta z \leq 14 m,
$$

la vitesse moyenne étant de l'ordre de $3500 \mathrm{~m} \cdot \mathrm{s}^{-1}$ et la fréquence centrale de $25 \mathrm{~Hz}$.

La méthode d'approximation numérique utilisée est la méthode des différences finies d'ordre 2 . Un schéma de Yee adapté aux PML est employé. Les pas de discrétisation retenus sont $\Delta x=12 m$ et $\Delta z=8 m$, une précision plus fine étant utilisée pour les variations verticales de l'impédance. Il reste à fixer le pas de discrétisation en temps. La condition CFL pour un milieu homogène, pour éviter le phénomène de dispersion du schéma de Yee, est la suivante

$$
c_{\max } \Delta t \sqrt{\frac{1}{\Delta x^{2}}+\frac{1}{\Delta z^{2}}} \leq 1
$$

avec $c_{\max }=\sup _{x, z} c(x, z)$. Dans le cas considéré, $c_{\max }=6013 \mathrm{~m} \cdot \mathrm{s}^{-1}$, ce qui implique $\Delta t \leq 1.1 \times 10^{-3}$. Cependant, les test numériques effectués indiquent que dans le cas de milieux hétérogènes, la condition de stabilité est inférieure au cas de milieux homogènes. Le pas de discrétisation retenu est finalement $\Delta t=10^{-3} \mathrm{~s}$.

\subsection{Figures résultats}

Dans chaque cas, on représente à gauche le champ de vitesse verticale dans le milieu demi-espace, au centre le champ de vitesse verticale dans un milieu borné par la PML, à droite l'erreur commise $u_{z e r r}(x, z, t)$, au temps $t=0.8 s$.

$$
u_{z e r r}(x, z, t)=\left|u_{z \infty}(x, z, t)-u_{z P M L}(x, z, t)\right| .
$$

La figure 2 correspond à un modèle homogène et la figure 3 correspond à un modèle bicouche. L'épaisseur de la couche PML est fixée à 20 points de discrétisation. L'échelle de l'erreur sélève pour chacun des deux cas à $10^{-5}$, pour un signal dont l'intensité est de l'ordre de $10^{-3}$, soit environ $1 \%$ d'erreur en norme $L^{\infty}$.

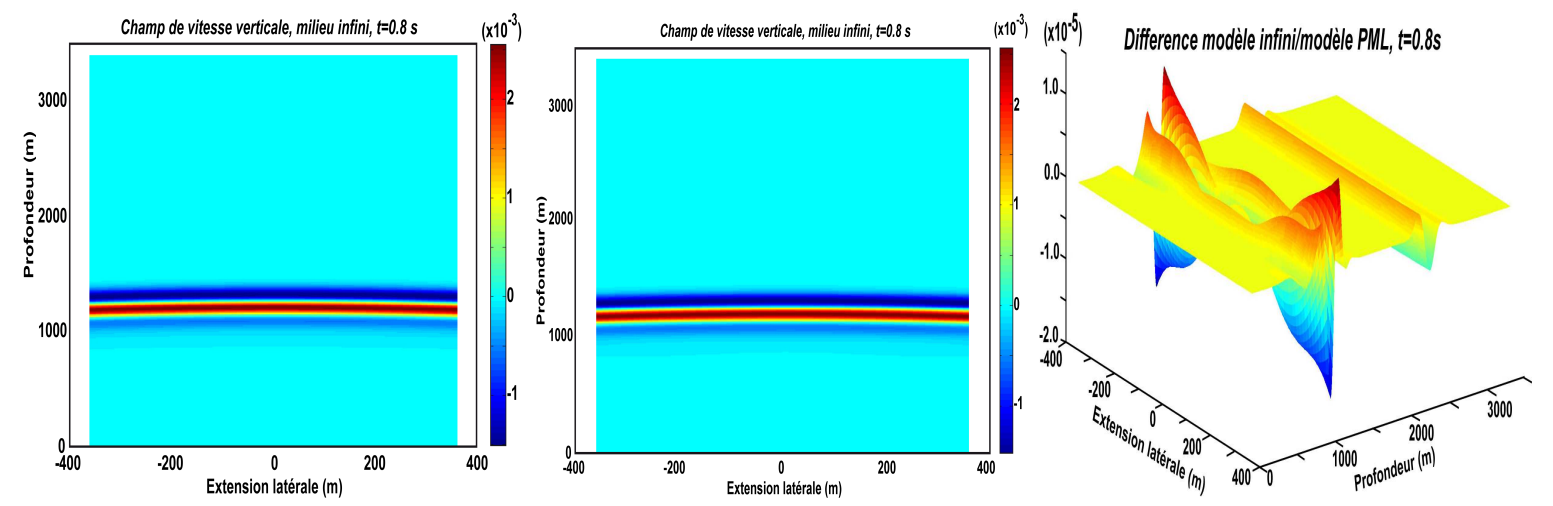

Figure 2. Champ de vitesse verticale pour un modèle homogène, à $t=0.8 \mathrm{~s}, 20$ points dans la PML

Le cas du modèle hétérogène (figure 1) est représenté figure 4. L'erreur augmente de manière significative: pour un signal d'intensité d'ordre $2 \times 10^{-3}$, l'erreur commise est d'ordre $2 \times 10^{-4}$, soit $10 \%$ d'erreur en norme $L^{\infty}$. Cependant, en augmentant la taille des couches PML à 40 points de discrétisation, l'erreur dimininue 

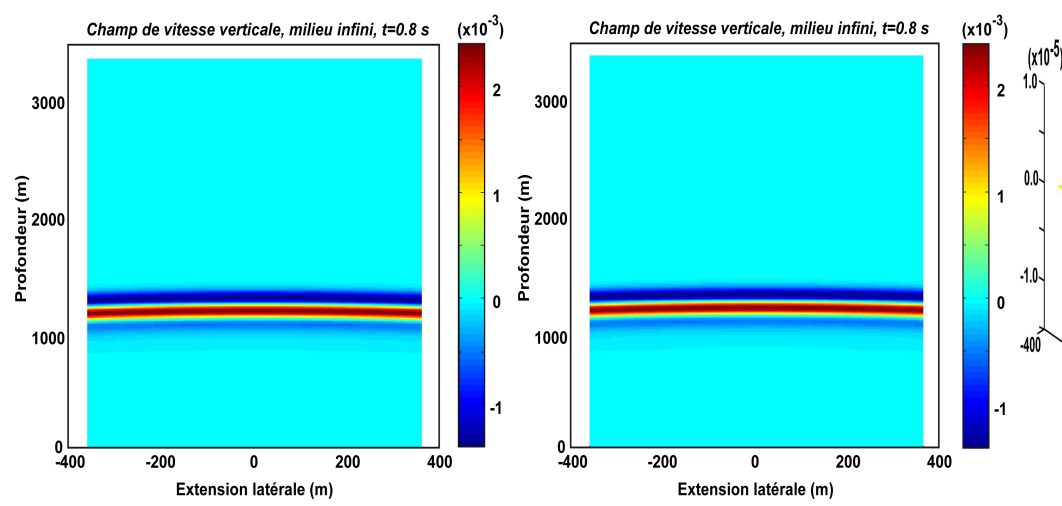

\section{Difference modèle infinilmodele PML, $t=0.8 \mathrm{~s}$}
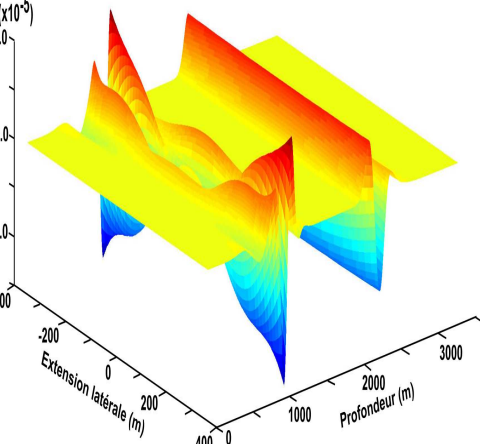

Figure 3. Champ de vitesse verticale pour un modèle bicouche, à $t=0.8 \mathrm{~s}, 20$ points dans la PML

signifiactivement (de l'ordre de $5 \%$ en norme $L^{\infty}$ ), et reste localisée à la frontière avec la couche PML (figure $5)$.
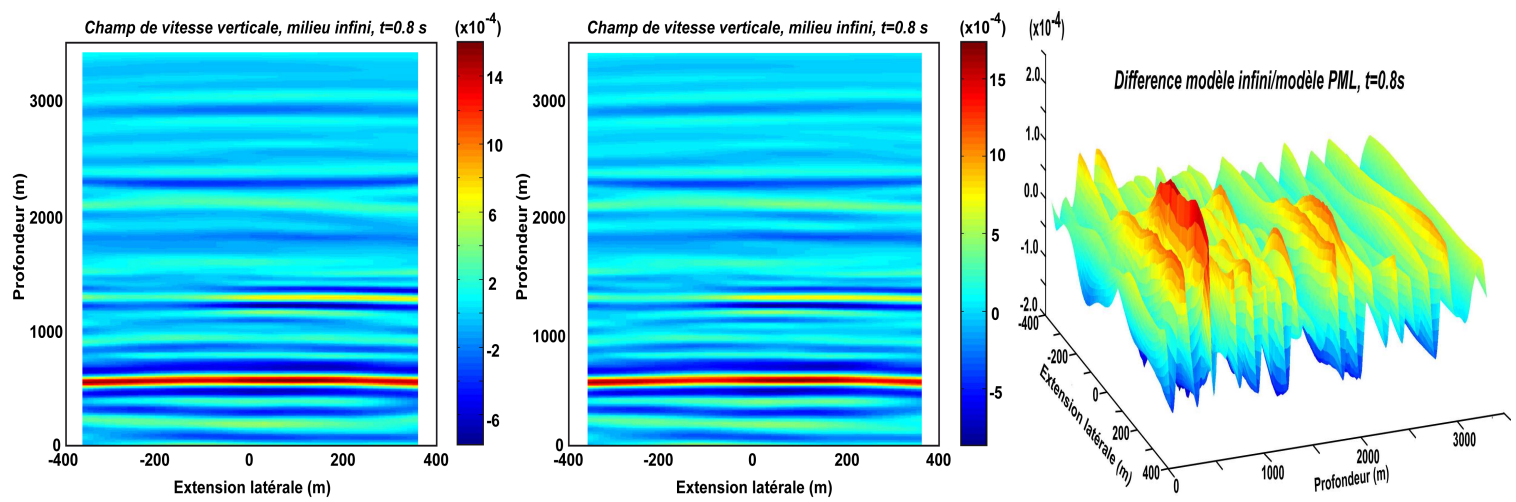

FiguRE 4. Champ de vitesse verticale pour le modèle hétérogène, à $t=0.8 \mathrm{~s}, 20$ points dans la PML

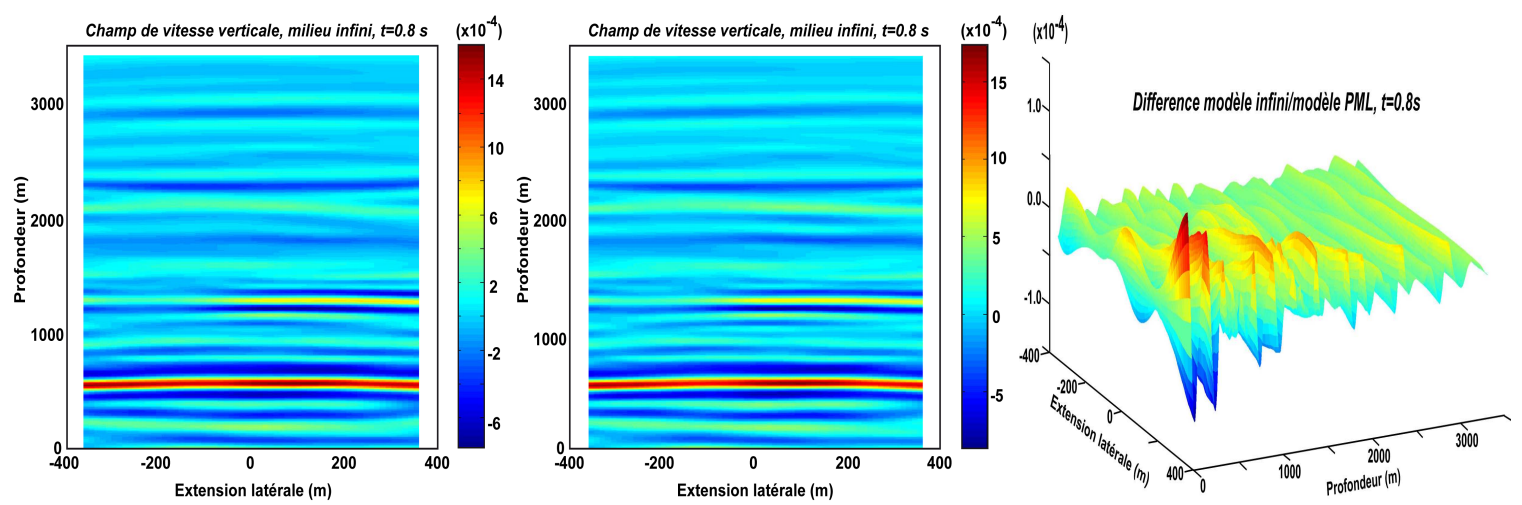

Figure 5. Champ de vitesse verticale pour le modèle hétérogène, à $t=0.8 \mathrm{~s}, 40$ points dans la PML 


\subsection{Conclusion}

Ces expériences montrent que la présence de fortes hétérogénéités dans le milieu de propagation perturbe le fonctionnement de la couche PML. Cependant l'augmentation de sa taille permet de réduire l'erreur d'approximation commise. De plus, il semble que celle-ci reste localisée au bord. Une étude plus précise de la localisation de l'erreur pourrait être envisagée. Afin d'améliorer ces résultats, l'introduction de conditions aux limites absorbantes à l'extrémité des couches PML pourrait également être testée, en remplacement des conditions de Dirichlet homogènes qui sont pour l'instant utilisées. La comparaison avec d'autres formulations des équations PML seraient également intéressantes. Beaucoup de travaux ont en effet été menés, tant sur les équations de Maxwell que sur les équations d'Euler, dont le but est de proposer de nouvelles formulations des équations PML [Nataf, 2006] [Rahmouni, 2004]. Un autre problème posé par la formulation de Bérenger utilisée ici est la décomposition des variables, qui augmente les coûts de calculs par l'introduction d'une équation supplémentaire. L'utilisation d'autres formulations sans décomposition pourrait permettre de surmonter cet inconvénient. D'autre part, la taille de la couche PML utilisée ici devenant importante, il serait également intéressant de comparer les résultats obtenus sur ce modèle avec ceux obtenus en utilisant des conditions aux limites transparentes. Les coûts de calcul augmentent en effet avec la taille de la couche PML, et il est possible que pour la même précision les conditions aux limites transparentes se révèlent moins coûteuses.

\section{REFERENCES}

[Abarbanel, Gottlieb, 1997] S.Abarbanel, D.Gottlieb, 1997, A mathematical analysis of the PML method, J. Comput. Phys., 134, pp. 357-363

[Bamberger, 1978] A.Bamberger, 1978, thèse de doctorat es-sciences mathématiques, Analyse, contrôle et identification de certains systèmes.

[Bamberger, Chavent, Lailly, 1979] A.Bamberger, G.Chavent, P.Lailly, 1979, About the stability of the inverse problem in 1-d wave equations - application to the interpreation of seismic profiles. Appl. Math. Optim. 5, 1-47.

[Bécache, Joly, 2002] E.Bécache, P.Joly, On the analysis of Bérenger's perfectly matched layers for Maxwell's equations, ESSAIM : M2AN, Vol. 36, 2002, pp. 87-119.

[Bérenger,1993] J.P.Bérenger, 1993, A Perfectly Matched Layer for the Absorption of Electromagnetic Waves, Journal of Computational Physics 114, 185-200.

[Bonnet, 2006] M.Bonnet, Problèmes inverses, Cours de master recherche à l'école centrale de Paris mention Matière, Structures, Fluides, Rayonnement, Spécialité Dynamique des structures et Systèmes couplés, 2006.

[Delprat-Jannaud, Lailly, 2004] F.Delprat-Jannaud, P.Lailly, 2004, The insidious effects of fine-scale heterogeneity in reflections seismology, Journal of seismic exploration, 13, 39-84 (2004).

[Delprat-Jannaud, Lailly, 2005] F.Delprat-Jannaud, P.Lailly, 2005,A fundamental limitation for the reconstruction of impedance profiles from seismic data, Geophysics, $\mathbf{7 0}, \mathrm{n} 1, \mathrm{R} 1-\mathrm{R} 14$.

[Hu, 1996] F.Q.Hu, 1996, On absorbing boundary conditions for linearized Euler equations by a perfectly matched layer, J.Comput.Phys. 129(1996)

[Kreiss, Lorenz, 2004] H.O.Kreiss, J.Lorenz, 2004, Initial-Boundary Value Problems and the Navier-Stokes Equations,SIAM, initial edition published by Academic Press, 1989, ISBN 0-89871-565-2.

[Macé, Lailly, 1986] D.Mace, P.Lailly, 1986, Solution of the VSP one-dimensional inverse problem : Geophysical Prospecting 34, 1002-1021.

[Métral, Vacus, 1999] J.Métral, O.Vacus, 1999, Caractère bien posé du problème de Cauchy pour le système de Bérenger, C.R. Acad. Sci. Paris Sér.I Math., 10, 847-852

[Nataf, 2006] F.Nataf, 2006, A new construction of perfectly matched layers for the lineaerized Euler equations, J. Comput. Phys., 214 n2, 757-772, 2006.

[Rahmouni, 2004] A.Rahmouni, Un modèle PML bien posé pour les équation d'Euler linéarisées, C.R. Acad. Sci. Paris Sér. I Math., 331, 159-164, 2000.

[Petit-Bergez, 2006] S.Petit-Bergez, Problèmes faiblement bien posés : discrétisation et applications, 2006, thèse soutenue à l'université de Paris XIII, discipline Mathématiques appliquées. 